\title{
Channel Estimation using Modified Extended Kalman Filter Based Algorithm for Fading Channels
}

\author{
G.Rajender, T.Anil Kumar, K.Srinivasa Rao
}

\begin{abstract}
The manner towards describing the effect of the bodily channel at the records succession is known as the Channel estimation. The Channel estimation strategies offer low multifaceted nature and better execution and are successfully implemented in correspondence frameworks. Be that as it may, they may be likewise inefficient of statistics transmission considering they use making geared up arrangements to assess the channel, so right right right here; a method is utilized wherein the restrained duration of getting prepared grouping is transmitted. The Kalman based totally calculation which may be finished effectively for channel estimation method. The kalman based calculation channel estimator activates a massive addition in execution even as contrasted with the information simply estimator. This calculation furthermore permits us to count on the scenario of the framework in advance than the threshold is genuinely gotten. In this paper, the channel estimation is completed making use of Kalman based calculation to foresee the price determinations of the situation of framework. Additionally the all out symphonious twisting of the refreshed us of $a$ is determined and restricted inner a particular absolutely worth. The channel under concept is a Rayleigh blurring channel.
\end{abstract}

Key terms: Channel estimation, Kalman clear out, Rayleigh blurring, total Harmonic Distortion.

\section{INTRODUCTION}

Kalman primarily based totally calculation is applied on this paper for channel estimation. This method dreams conditions for the Kalman primarily based completely calculation, which can be clarified in [1]. Channel estimation is a huge idea on the equal time as we transmit flag thru a ways flung ways. On the off hazard that the channel is idea to be direct, the channel gauge is basically the gauge of the power response of the framework. It want to be driven all all over again that channel estimation is simplest a systematic portrayal of what is in reality going on. A tremendous channel gauge is one wherein a few form of blunder minimization requirements is fulfilled (as an instance MMSE). The technique of direct estimation is depicted in element in [2]. The facts given is a sine wave.

The strategies thru which amazing estimation techniques are associated with the direct are regarded inside the sign making geared up fields in [3] and [4]. The calculation is an expansion of the Kalman channel. So you could consider EKF, it's miles inescapable to understand Kalman channel, that is in [5]. The MATLAB executions of Kalman channel are in [6]. A correlation amongst Kalman primarily based definitely calculation and

Revised Manuscript Received on September 10, 2019.

G.Rajender, Assoc. Professor of ECE Dept., CMRIT, Hyderabad, Telangana, India.

T.Anil Kumar, Professor and Head of ECE Dept., CMRIT, Hyderabad, Telangana, India.

K.Srinivasa Rao, Professor of ECE Dept., Ellenki Engineering college, Hyderabad, Telangana, India.
Kalman channel is critical to realize the evaluation and contrasts to observe the particular one to be achieved for our motivation. This is clarified in [7], in which we've got got had been given the conditions for the channels.

The predicted gauges and refreshed checks are located the usage of kalman based absolutely calculation estimation. This method for estimation is for the most element implemented for the channel estimation and examination of non-immediately frameworks. Absolute Harmonic Distortion the various refreshed gauge and the actual state is also decided. Simply the symphonious mutilation or THD of a sign is an estimation of the consonant twisting gift inside the signal.

The channel under idea is a Rayleigh blurring channel. Rayleigh blurring is a sensible version at the identical time as there are numerous gadgets in the circumstance that burn up the radio signal earlier than it touches base on the collector.

The channel below concept is a Rayleigh blurring channel. Rayleigh blurring is a sensible model even as there are various articles inside the state of affairs that disperse the radio signal in advance than it touches base on the recipient [8].

\section{TERMINOLOGIES}

\section{A. Channel Estimation}

The method of channel estimation has been clarified. There are techniques for channel estimation. They're getting equipped grouping/pilot based totally totally truely channel estimation and visually impaired channel estimation [2]. Estimation calculations purpose limiting the recommend squared blunder.

We try and gauge $h$ inside the sight of clamor and version confuse, with the resource of way of in truth searching the channel yield $y(n)$ :

\section{$\mathrm{y}(\mathrm{n})=\mathrm{hT} * \mathrm{x}(\mathrm{n})+\mathrm{v}(\mathrm{n})$}

In the figure beneath, $\mathrm{e}(\mathrm{n})$ is the estimation mistake. The point of most channel estimation calculations is to limit the mean squared mistake (MMSE), E[e2(n)] while using as meager computational assets as conceivable in the estimation procedure. 


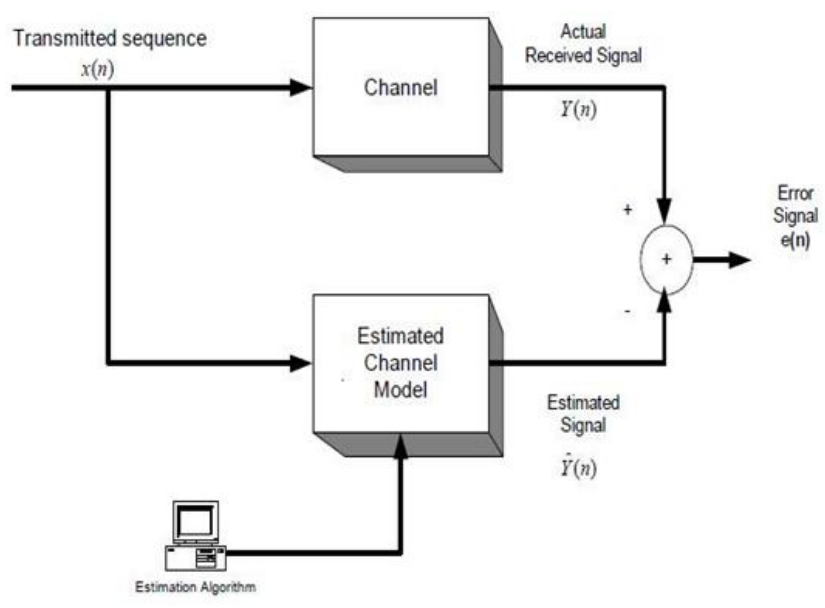

Figure 1. General Channel Estimation

While an estimation version has been built up, its parameters need to be constantly refreshed (assessed) that allows you to restriction the blunder due to the fact the channel adjustments. Inside the occasion that the recipient has from the earlier analyzing of the records being despatched over the channel, it can use this information to build up a specific gauge of the energy response of the channel. This technique is largely called making prepared grouping based Channel estimation. It has the gain of being utilized in any radio correspondences framework effectively.

Regardless of the reality that that is the maximum mainstream approach getting used in recent times, regardless it has its dangers. One of the conspicuous downsides is that it's far inefficient of switch speed. Precious bits in a casing which can were commonly used to move information are loaded down with making equipped successions for channel estimation. This method likewise endures because of the manner that maximum correspondence frameworks ship records lumped outlines. It's miles genuinely after the receipt of the complete trouble that the channel gauge may be separated from the installation making geared up grouping. For short blurring channels this likely won't be remarkable due to the fact the rationality time of the channel may be shorter than the casing time. Parameters are significant as a proportion of the individual of estimation. They're predisposition and fluctuation of estimation. A predisposition (obscure) implies a perpetual (un-detachable) mistake inside the assessments. A trustworthy-minded estimator is attractive. Fluctuation of estimations gives the capability degree of deviation of the estimator from the real incentive to be assessed. An estimator with a low fluctuation (in a super worldwide least) is appealing. Additionally, a right away estimator is appealing because of the simplicity of execution. For that reason, a at once sincere-minded estimator with least trade is relatively fascinating.

The pilot based totally channel estimation is of kinds. They're the square type and the comb kind. In the square type, all subcarriers in the casing are pilot tones. Likewise, the rims are transmitted sometimes. It makes use of a similar divert estimation for entire For the situation of brush type, simply part of the subcarriers within the casing are pilot tones.

Dazzle techniques rather require no training preparations. They use sure important numerical data about the shape of records being transmitted. These strategies can be transmission capability efficient but have their very private downsides. They may be famously no longer on time to combine. Their distinct drawback is that the ones techniques are amazingly computationally critical and as a result are unreasonable to execute continuously frameworks. They likewise do not have the compactness of making equipped grouping primarily based totally absolutely strategies. One calculation that works for a selected framework might not art work with a few different due to the truth they deliver numerous types of records over the channel.

\section{B. Kalman based calculation}

The Kalman channel, otherwise called immediately quadratic estimation (LQE), is a calculation that uses a development of estimations observed after a while, containing commotion (unusual sorts) and unique errors, and produces value determinations of tough to recognize factors so you can in famous be extra real than those relying on a solitary estimation by myself. All of the extra formally, the Kalman channel works recursively on floods of uproarious records to create a factually high-quality gauge of the fundamental framework united states.

The calculation works in a -increase system. In the expectation step, the Kalman channel produces charge determinations of the present u . S . A . Factors, along their vulnerabilities. Even as the stop end result of the following estimation (basically defiled with some degree of blunder, which encompass arbitrary clamor) is watched, the ones critiques are refreshed the use of a weighted ordinary, with greater weight being given to gauges with higher sureness.

Most right frameworks are non-right away. The Kalman filter for nonlinear fashions is indicated the Kalman based totally clear out whilst you take into account that it is an allencompassing utilization of the first Kalman filter. Kalman based filter (KF) uses non-straight away fashions of each the approach and notion models even as the Kalman filter (KF) uses direct fashions [1].

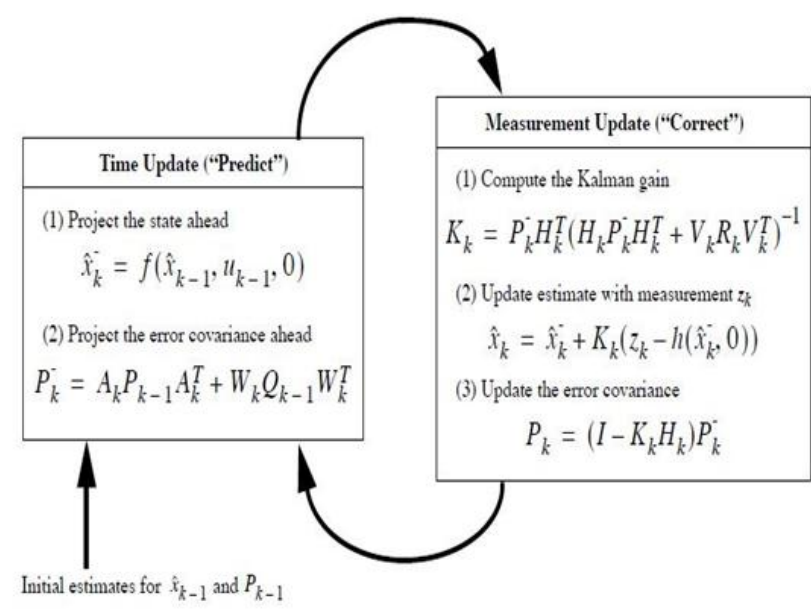

Figure 2. Kalman Filter Prediction Estimation Cycle 
Fig 2 indicates the Kalman based totally truly filter Prediction Estimation Cycle which offers situations to do the estimation approach. The Kalman primarily based completely channel (KF) offers a guess of the proper gauge. The non-linearity of the framework's factors are approximated through a linearized shape of the non-at once framework version across the ultimate country gauge. For this wager to be great, this linearization should be a terrific estimation of the non-direct model in the complete vulnerability area associated with the state gauge

\section{Rayleigh Fading}

Rayleigh blurring is the most considerable type of blurring that takes vicinity in the city situations. It's miles a true model for the impact of a ramification region on a radio signal, for instance, that used by a long way off gadgets. Rayleigh blurring models take transport of that the size of a sign that has prolonged beyond via the form of transmission medium (moreover called a correspondence channel) will range haphazardly, or blur, as indicated via a Rayleigh dissemination [8].

As a ways as possible hypothesis holds that, if there is adequately plenty deplete, the channel energy response can be properly-tested as a Gaussian manner impartial of the dispersion of the character additives. Inside the occasion that there's no winning phase to the disperse, at that element the form of way will have 0 suggest and degree uniformly appropriated between zero what's extra, $2 \pi$ radians. The envelope of the channel response will alongside the ones traces be Rayleigh appropriated. Rayleigh blurring is a touch scale impact. There is probably mass homes of the earth, for instance, manner misfortune and shadowing whereupon the blurring is superimposed.

Besides there can be a whole lot of gadgets throughout the at once manner. The ones devices also can serve to mirror, refract, and so on the signal. Consequently, there are numerous unique strategies with the aid of which the signal may also moreover arrive at the recipient. On the factor while the sign arrive on the recipient, the overall sign is a mixture of the huge variety of signal which have arrived at the collector through the big quantity of severa strategies which can be available. The ones signal will all mixture collectively, the period of the sign being big. Subordinate upon the manner thru way of which the ones signal usual collectively, the sign will alternate in terrific. Within the event that they have been all in diploma with each other, they may all embody. Besides this isn't always in maximum instances the situation, as some is probably in take away and others of degree, contingent at the one of a type way lengths, and therefore a few will in elegant upload to the overall sign, regardless of the fact that others will subtract. This systems the Rayleigh circulate for the channel.

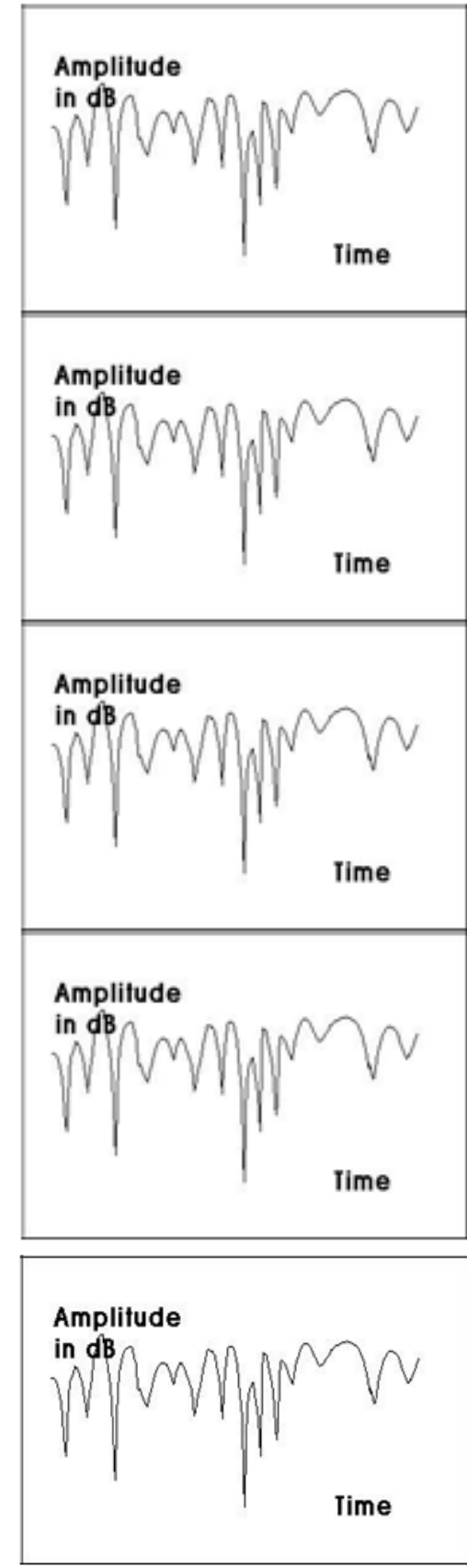

Figure 3. A sample of a Rayleigh fading signal.

$$
\begin{aligned}
& \text { where } \quad \begin{array}{c}
p_{R}(r)=\frac{2 r}{\Omega} e^{-r^{2} / \Omega}, r \geq 0 \\
\Omega=E\left(R^{2}\right)
\end{array}
\end{aligned}
$$

\section{Harmonic Distortion}

The entire consonant bending is characterised as the share of everything of the forces of every single symphonious section to the intensity of the essential frequency. THD is achieved to portray the linearity of sound frameworks and the power nature of electrical power frameworks. At the component on the same time because the facts is an unadulterated sine wave, the estimation is most normally the share of the complete of the forces of all better consonant 
frequencies to the strength at the precept symphonious, or maximum essential frequency.

THD $(\%)=$ sqrt (consonant electricity)/sqrt (signal electricity)

THD=harmonic manipulate/signal energy

THD is likewise normally characterized as a sufficiency percent in preference to a power percentage, bringing approximately a this means that of THD that's the rectangular basis of that given above. The all out consonant contortion, or THD, of a signal is an estimation of the symphonious mutilation present and is characterized as the proportion of the combination of the forces of every unmarried symphonious issue to the intensity of the important frequency.

\section{DEVELOPED APPROACH \& RESULTS}

In this paper, the modified extended Kalman clear out is used for channel estimation. The information given as sine wave. The Rayleigh channel is used . Via the usage of then the foreseen measures and revived checks are to be found. The whole scale consonant twisting the various invigorated test and the actual kingdom in like manner have to be resolved. The invigorated state is usually called the assessed u. S ..

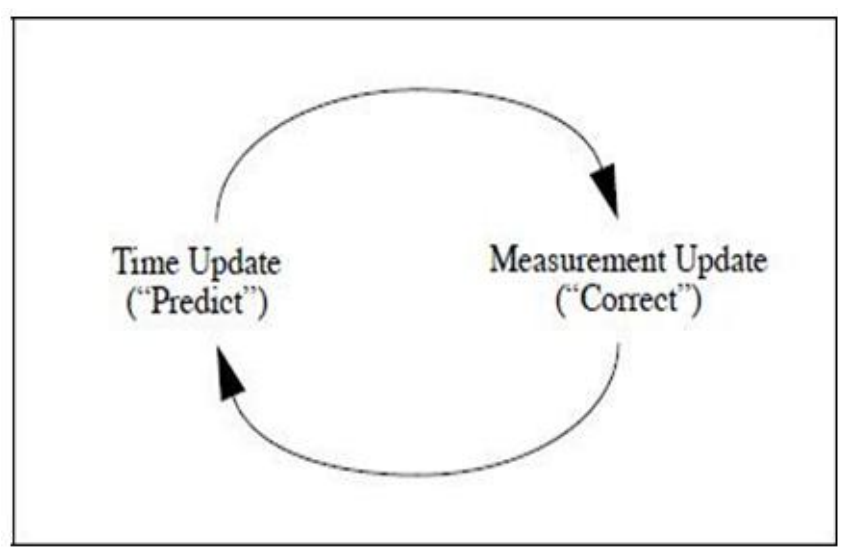

Figure 4. Kalman Filter Cycle

\section{A. Flowchart}

The flowchart consists of the unique ranges of the Kalman channel direct estimation applied inside the challenge completed. The foremost stage is the presumption of a correspondence channel for the transmission. Proper right here a Rayleigh blurring channel is considered. At that detail the underlying parameters for the estimation are popular with the purpose that the expectancy and updation in estimation can be all began.

The estimation technique is started and the refreshed and predicted reviews are located. The errors and without a doubt the consonant contortion are decided at that factor. For the reason that THD for regular house hold motives for present is below three\%. In any case, because of blurring the mentioned blurring is $15 \%$. So whilst THD progresses within the route of becoming in the $15 \%$ truly worth, at that trouble the cycle is therefore ceased and the charts are plotted.

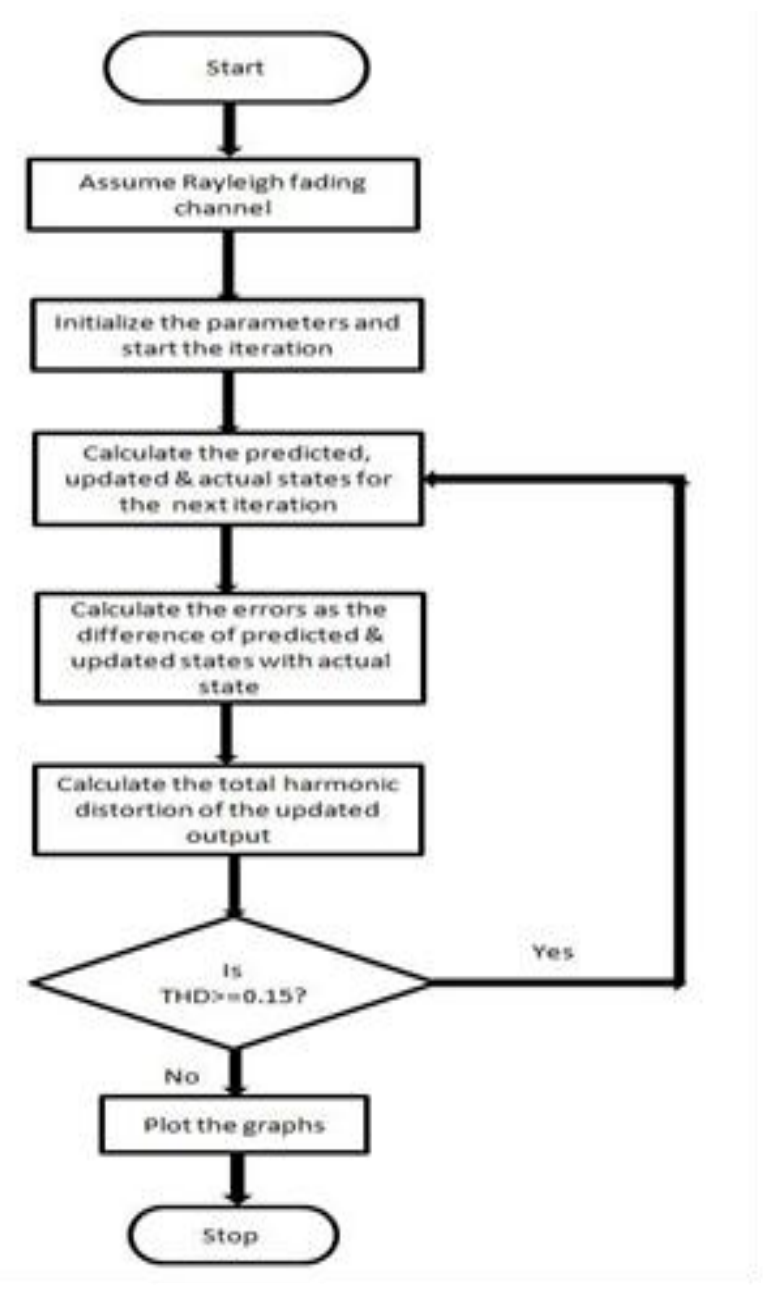

Figure 5. Flowchart

\section{B Algorithm}

1. Begin

2. Accept the channel with Rayleigh blurring.

3. Introduce the accompanying parameters: range of emphasess $(\mathrm{N})$, nation mistake $(\mathrm{W})$, estimation blunder $(\mathrm{V})$, estimation country $(\mathrm{H})$, method commotion covariance $(\mathrm{Q})$, estimation commotion covariance (R), refreshed evaluations, anticipated value determinations, real country (x), Kalman advantage $(\mathrm{k})$

4. Calculate updated and predicted states and error covariance appraises, the real state and Kalman advantage for $\mathrm{N}$ cycles utilising the EKF conditions as in [7].

5. find out the errors, error 1 and error 2 because the distinction

of refreshed and anticipated state with the real state

utilizing the situations given beneath. error $1=$ real kingdom - predicted country error $2=$ actual state - up to date state 6. locate the all out symphonious distortion(THD) of the refreshed sine yield as the percentage of overall of sounds capability to the key energy in both of the channels.

7. within the event that THD $>=0.15$, go to level four. Else go to level eight

8. Plot the diagrams.

9. cease 


\section{CONSEQUENCES \& RESULTS}

The actual state, revived state and foreseen kingdom were resolved. by using then the slip-up and THD similarly are resolved, the accentuation is ended at the same time as the THD come inner $20 \%$ that's ordinary properly worth.
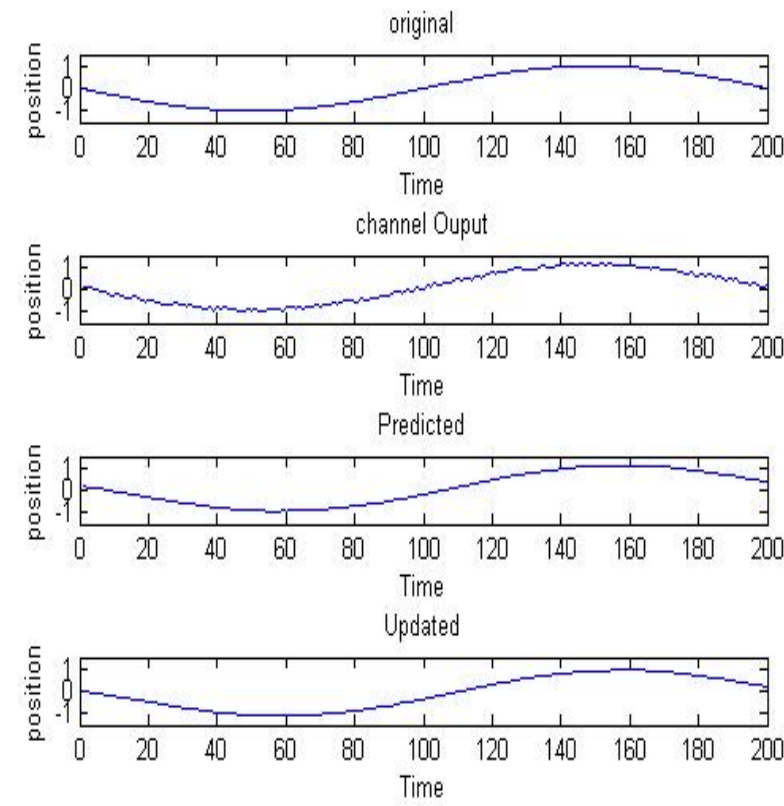

Determine 6. Actual kingdom, channel o/p country, anticipated country and up to date united states

the primary state is the real sine wave that's to be gotten in keeping with the situation of the Kalman primarily based absolutely clear out calculation. The expected state and the assessed/refreshed u. S . Are moreover regarded inside the determine 6.
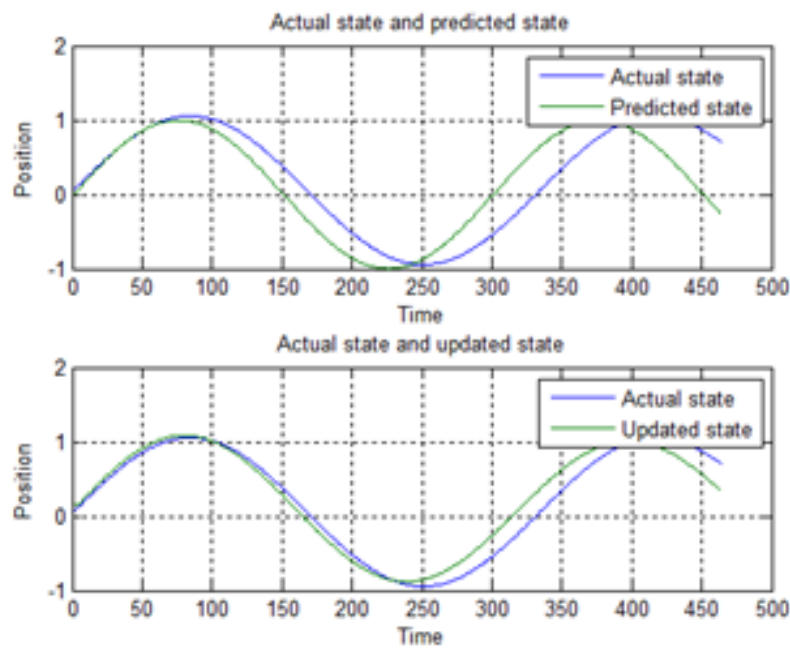

Determine .7The consolidated discern of the real, expected and Refreshed united states of the united states.

The chart 7 makes it workable to precisely discover the distinction present inside the middle of the actual u. S . A ., anticipated kingdom and the refreshed u. S .. We will see that there are mutilations for the predicted and refreshed sine wave yields contrasted with the real wave. The right wave is the real precise that need to be gotten on the beneficiary. The mutilations show the mistake or flaw related to the
Kalman primarily based totally calculation estimation technique
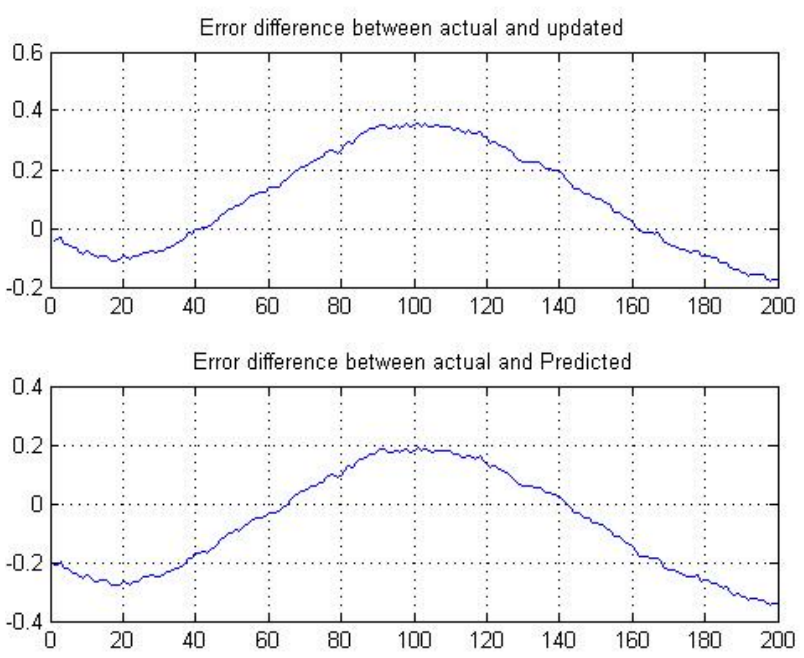

Figure eight. The mistake a number of the predicted and refreshed nation With the actual usa.

The determine eight demonstrates the error some of the real country and the anticipated nation, error 1 and the blunder among the real nation and the refreshed kingdom, error2 in a huge scale. From the chart, unmistakably the blunder of the Kalman based totally absolutely calculation estimation approach is similarly an awful lot a whole lot much less.

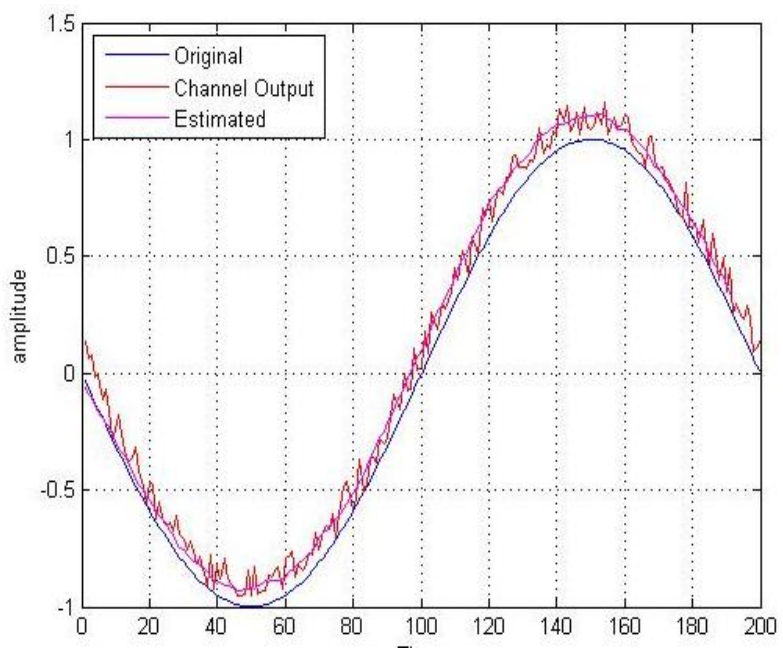

Figure 9. True signal Channel output, and envisioned $\mathbf{o} / \mathbf{p}$

The determine 9 demonstrates the first sine wave, channel yield and the assessed channel yield of the framework.

V. Conclusion The Kalman based absolutely calculation is a lot of numerical conditions that offers a skilled computational affiliation of the least-squares method. The channel is appreciably floor-breaking in some angles: it bolsters estimations of past, gift, or even future states, and it 
is able to do as such however even as the perfect concept of the installed framework is hard to apprehend.

The Kalman based totally calculation has been finished and have decided the contortion of the refreshed gauge sine wave. This has been done in a Rayleigh blurring state of affairs. The bending nicely properly well worth has been confined underneath $15 \%$ by using way of appearing severa style of cycles. The inducement inside the back of why I implemented the Kalman based channel is that the framework is nonlinear. On this manner, at a few component problem the framework is nonlinear we need to utilize the all-encompassing Kalman channel. Glaringly we can likewise linearize the framework around a working issue. In any case, the Kalman based totally channel has a advanced meeting than the Kalman channel, and is freed from preference of an hobby factor. For our state of affairs, the all out symphonious mutilation joined underneath $15 \%$ at the 464th emphasis of the estimation approach steady with the normal beginning characteristics considered in this approach.

The makes use of of the Kalman primarily based channel includes following a shifting item (gauge its region and tempo at whenever), anticipating that pace at modern time is velocity at past time in addition to Gaussian commotion). That is finished through making use of an association of place perceptions coming in consecutively. Destiny adjustments are viable for moving the info wave from sine wave to particular square wave, triangular wave and so forth. Additionally a changed prolonged Kalman clean out may be applied to reveal signs of development execution.

\section{REFERENCES}

1. Gabriel Terejanu, prolonged Kalman, department of laptop science \& Engineering, university at Buffalo, big apple 14260.

2. Rupul Safaya, "A Multipath Channel Estimation algorithm the usage of a Kalman clear out"

3. Steven M. Kay, basics of Statistical signal Processing, quantity I: Estimation concept (v. 1).

4. Vincent terrible, An creation to sign Detection and Estimation.

5. Greg Welch \& Gary Bishop, "An advent to Kalman filter out" , TR ninety five-041,department of laptop technological understanding, university of North Carolina, Chapel Hill, Chapel Hill- NC-27599-3175, 24 July, 2006.

6. Rachel Kleinbauer, "Kalman filtering implementation with MATLAB", university at Stuttgart.

7. Maria Isabel Ribeiro, "Kalman and prolonged Kalman Filters: concept, Derivation and houses", Institute for structures and Robotics.

8. T.S. Rappaport, wireless Communications requirements and exercising.. Creator: Prentice corridor press. 1996. 